\title{
CORRECTION
}

\section{Correction to: Can PET/CT be used more effectively in pleural effusion evaluation?}

\author{
Fikri Selcuk Simsek ${ }^{1} \cdot$ Dogangun Yuksel $^{1}$ - Olga Yaylali ${ }^{1} \cdot$ Halil Serdar Aslan $^{2} \cdot$ Emel Kılıçarslan $^{3} \cdot$ Ferda Bir $^{3}$. \\ Muhammet Arslan ${ }^{2} \cdot$ Fatma Ezgi Can ${ }^{4} \cdot$ Erhan Ugurlu $^{5}$
}

Published online: 5 August 2021

(c) Japan Radiological Society 2021

\section{Correction to: Japanese Journal of Radiology https://doi.org/10.1007/s11604-021-01155-z}

In Results heading of Abstract section, the sentence:

"However, if PE maximum standardized uptake value (SUV$\max )>1.3$ or PE SUV $\mathrm{max}_{\text {mean }}$ standardized"

Should read as:

"However, if PE maximum standardized uptake value (SUV$\max )>1.3$ or $\mathrm{PE} \mathrm{SUV}_{\max } /$ mean standardized"

The original article has been updated.

The original article can be found online at https://doi.org/10.1007/ s11604-021-01155-z.

Fikri Selcuk Simsek

fselcuksimsek@gmail.com

1 Nuclear Medicine Department, Faculty of Medicine, Pamukkale University, Denizli, Turkey

2 Radiology Department, Faculty of Medicine, Pamukkale University, Denizli, Turkey

3 Pathology Department, Faculty of Medicine, Pamukkale University, Denizli, Turkey

4 Biostatistics Department, Faculty of Medicine, Izmir Katip Celebi University, Izmir, Turkey

5 Pulmonology Department, Faculty of Medicine, Pamukkale University, Denizli, Turkey
The word "mean" should be in online and not in subscript as published.

Publisher's Note Springer Nature remains neutral with regard to jurisdictional claims in published maps and institutional affiliations. 